\title{
Onset of Elasto-capillary Bundling of Micropillar Arrays: A Direct Visualization
}

\author{
Diana Garcia-Gonzalez, Jacco Snoeijer, Michael Kappl, and Hans-Jürgen Butt*
}

Cite This: Langmuir 2020, 36, 11581-11588

Read Online

ABSTRACT: When a liquid drop gets into contact with a soft array of microstructures, capillary forces at the three-phase contact line can lead to critical deformations. Microstructures may collapse and form bundles or even patterns. So far, viewing the kinetics of bundling at the menisci scale has remained elusive. Here, we use laser scanning confocal microscopy to directly image the menisci between micropillars. We image structural changes in polydimethylsiloxane micropillar arrays during the Cassie-to-Wenzel transitions of a water drop evaporating on top of the array. We demonstrate how the regular pillar array undergoes a spontaneous symmetry breaking as the first step to the formation of pillar

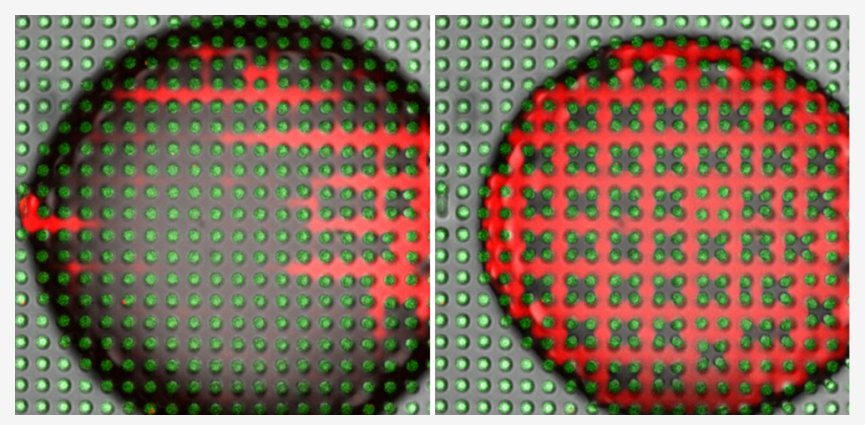
bundles. A comparison of the Cassie-to-Wenzel transition in air and FC40 indicates that the local contact angle determines the outcome of the bundling process. Based on these observations, we develop a simple model using the local contact angle, stiffness of the pillars, and interfacial tension of the liquid to predict the onset of the symmetry breaking.

\section{INTRODUCTION}

Capillary forces play a role in diverse natural settings. Usually, capillary forces have more impact on the small scale, for example, when a water drop sits on a so-called superhydrophobic surface. A good model for superhydrophobic surfaces is an array of hydrophobic micrometric pillars. ${ }^{1,2}$ In this scenario, one encounters the fact that the drop remains spherical as it is dominated by capillary forces that allow it to minimize its surface energy and thus not wet the surface (Cassie-Baxter state ${ }^{3}$ ). However, if the drop wets the array of micrometric pillars (Wenzel state), the pillars in the array may bend and collapse upon draining or evaporation of the liquid. $^{4-7}$ The response of the pillars depends on their height and material as well as the surface tension of the drop. ${ }^{8,9}$

The collapse of the pillars is analogous to a paintbrush dipped in liquid. ${ }^{10-14}$ When the brush is pulled out of the liquid, its bristles aggregate because of the interplay between the capillary and elastic forces. The elastic forces, directly related to the material properties of the solid, try to straighten the bent bristles. In contrast, capillary forces pull the bristles together. This joint effect is called elasto-capillarity and has been studied extensively ${ }^{14,15}$ in a wide range of scenarios, from clumps of wet bristles, ${ }^{16}$ feathers, ${ }^{12}$ or hairs ${ }^{17}$ to carbon nanotubes. $^{18}$

Elasto-capillarity is of particular importance for the fabrication of micro-electro-mechanical systems (MEMS) where wet lithography is used to produce micrometric structures of controllable size and order. In wet lithography, the last step involves rinsing the sample with a developing agent. This step is both vital for the structures to be finalized and very delicate for slender structures, like pillars, since the pattern can collapse when draining the developing agent. ${ }^{4}$ Once the structures have collapsed, sticking occurs due to adhesion forces, which render the process irreversible in most cases. Uncontrolled structural collapse is not desirable as it damages the quality of the MEMS. However, a controlled collapse can lead to patterns of self-assembled bundles of different sizes and shapes. ${ }^{16}$ In fact, the elasto-capillary interactions in the lithography processes open up the possibility of producing higher-aspect ratio structures and even three-dimensional lithography. ${ }^{18,19}$ So far, it has been possible to control the clustering process by varying different parameters like the geometry of the sample and its tilting angle when evaporating a fluid ${ }^{20}$ or, at the nanometric scale, by locally varying the relative positions between pillars. ${ }^{21}$

A global fabrication strategy is still obscured by the lack of information regarding the onset of the process at the scale of microstructures. Directly observing the capillary menisci between the pillars is key to understand the onset of the bundling process, yet in this context, it has only been done by

Received: July 21, 2020

Revised: September 7, 2020

Published: September 8, 2020 
using top-view images. ${ }^{9,20,22,23}$ These images show the movement and final position of the top of the structures. Such observations are valuable to classify the different bundling geometries but do not capture the shape of the menisci, which ultimately determines the capillary force applied on each pillar. To resolve this, previous studies have focused on setups that are conceptually simpler than micropillar arrays, for example, a beam piercing a liquid surface, ${ }^{6}$ the multiple states of equilibrium of a pair of beams holding fluid in between, ${ }^{24}$ or the capillary rise between two slender lamellae. ${ }^{11,13}$ There have also been many studies regarding the agglomeration of multiple high-aspect ratio structures like the case of multiple lamellae forced to pierce a liquid's interface ${ }^{25}$ and numerical studies where they used multiple spring block elements to model the cluster size and dynamics on a two-dimensional system. ${ }^{7,26}$ To our knowledge, however, time-resolved images of the menisci in micropillar arrays during the entire process of bundling, have remained elusive.

In this paper, we directly image the onset of the threedimensional self-assembly of micropillars. We image both topview and cross-sectional videos of the process by using a laser scanning confocal microscope (LSCM). We acquire critical information regarding the time-resolved shape of the interpillar meniscus between the micrometric array of soft pillars. In particular, we show how the menisci evolve during the bundling process. Our work focuses on the onset of the bundling where the initially regular pillar array undergoes a symmetry breaking. By comparing pillar arrays filled with air or with FC-40, it is found that the symmetry breaking of the pillar array is sensitive to the contact angle of the menisci on the pillars. We devise a simple two-dimensional model in which we incorporate elasticity and capillarity effects. While previous studies focus on the conditions necessary for bundles to stick, ${ }^{10,17}$ we consider the initial breaking of symmetry of the pillar array. By comparing the results of the model to the experiment, we confirm the importance of the contact angle.

\section{EXPERIMENTAL SECTION}

Sample Preparation. Polydimethylsiloxane (PDMS) pillar arrays were patterned onto a $170 \mu \mathrm{m}$-thick glass slide. The final arrays were superhydrophobic due to the combination of structure and hydrophobicity from the PDMS. The typical apparent advancing and receding contact angles of the surface were measured by moving the drop on the surface and observing the contact angle change via LSCM. The values were $150^{\circ}$ and $140^{\circ}$. The cylindrical pillars, ordered in a square grid, had the following dimensions: height, $h=40$ $\mu \mathrm{m}$, diameter, $d=20 \mu \mathrm{m}$, and center-to-center spacing between pillars, $w=40 \mu \mathrm{m}$.

The pillars were fabricated via a two-step photolithography process. ${ }^{27}$ First, a template was prepared by exposing a spin-coated layer of negative SU-8 photoresist on a $1 \mathrm{~mm}$-thick glass slide to UV light through a chromium mask with circular holes. The sample was rinsed with the developer solution (Microchem) followed by isopropyl alcohol and dried under a stream of nitrogen gas. The resulting template with the SU-8 pillar structure was silanized for $1 \mathrm{~h}$ with $1 \mathrm{H}, 1 \mathrm{H}, 2 \mathrm{H}, 2 \mathrm{H}$-perfluorooctyl-trichlorosilane via chemical vapor deposition under vacuum. The template was used to create a negative elastomeric stamp of the target structure. For that, PDMS (crosslinker to base ratio, 1:10) was poured on the SU-8 template and crosslinked in an oven at $150{ }^{\circ} \mathrm{C}$ for $1 \mathrm{~h}$. The PDMS stamp (with holes) was mechanically detached from the SU-8 template; the silane layer helped release the PDMS structure from the SU-8 template.

Next, the PDMS stamp was silanized with $1 \mathrm{H}, 1 \mathrm{H}, 2 \mathrm{H}, 2 \mathrm{H}$ perfluorooctyl trichlorosilane via chemical vapor deposition. A new mixture of PDMS (cross-linker to base ratio, 1:10) was fluorescently labeled with Coumarin $6(0.04 \mathrm{mg} / \mathrm{mL})$ for imaging later by confocal microscopy. The stained PDMS was spin-coated onto a $170 \mu \mathrm{m}$-thick glass slide (no. $1.5 \mathrm{H}$ from Marienfeld Superior). The PDMS-coated glass substrate was pressed into contact with the silanized PDMS stamp and placed into a vacuum chamber to remove any residual air pockets and ensure complete filling. The stained PDMS was then cross-linked at $60{ }^{\circ} \mathrm{C}$ for $15 \mathrm{~h}$. Finally, the stamp and the sample were mechanically separated from each other by peeling.

A key element in this study is the flexibility of the pillars. Using beam theory, one obtains a linear relation between the pillar deflection $x$ and a horizontal point force $F_{x}$ applied at the end of the pillar ${ }^{28}$

$$
F_{x}=\frac{3 \pi E d^{4}}{64 h^{3}} x
$$

as was validated experimentally by Papadopoulos et al. ${ }^{29}$ Thus, the pillars react to a horizontal end load as a linear spring with a spring constant of $k=\frac{3 \pi E d^{4}}{64 h^{3}}$. Here, the Young's modulus of the PDMS substrate, $E=0.54 \pm 0.04 \mathrm{MPa}$, was determined using a home-built indenter system in which a $2 \mathrm{~mm}$ radius ruby sphere was used as an indenter. With these parameters, the elasto-capillary length ${ }^{14}$ $\left(\sim \sqrt{E r^{3} / 8 \gamma}\right)$ is approximately $36 \mu \mathrm{m}$, which is comparable to the pillar height so that pillar deformations can be expected. On the contrary, the pillars are not expected to bend due to the pressure force within a $4 \mu \mathrm{L}$ drop since the pressure force is around 2 orders of magnitude smaller as compared to Euler's critical load for our pillars $(8 \mu \mathrm{N})$.

To measure the Young's modulus, the sample was placed on a glass slide that was mounted on top of a force sensor (GSO10 load cell, Transducer Techniques, Temecula, CA). The ruby sphere was connected to a piezo translation stage with a $1500 \mu \mathrm{m}$ movement range (P-629.1CD, Physik Instrumente, Karlsruhe, Germany). The sphere was moved toward the sample with a linear motion at a speed of $10 \mu \mathrm{m} / \mathrm{s}$ until a predefined contact force of $5-10 \mathrm{mN}$ was reached. The movement of the piezo stage was then reversed until complete separation was achieved again. Both the piezo position and force were recorded by a computer equipped with an $\mathrm{AD}$ board (NI6052, National Instruments, Austin, TX). The force versus indentation curves obtained were fitted using the JKR model ${ }^{30,31}$ to determine the Young's modulus of the samples. Assuming a PDMS Poisson ratio of 0.5 , a work of adhesion of $W_{\mathrm{a}}=0.10 \pm 0.01 \mathrm{~J} / \mathrm{m}^{2}$ was obtained.

Experimental Data Acquisition and Analysis. For all measurements, we used drops of ultrapure (Milli-Q) water dyed with Alexa 647 at a concentration of $0.1 \mathrm{mg} / \mathrm{mL}$. The surface tension of the Alexa-dyed water was $65.4 \pm 0.3 \mathrm{mN} / \mathrm{m}$ as measured using a Wilhelmy plate. In part of the experiments, the PDMS pillars were infused with FC-40, a fluorinated oil with a surface tension of $14.2 \pm$ $0.3 \mathrm{mN} / \mathrm{m}$. FC-40 was used because it combines index matching (see below) with low water solubility $(7 \mathrm{ppm})$. The interfacial tension between FC-40 and water is $52.1 \pm 0.2 \mathrm{mN} / \mathrm{m}$, and FC-40 does not swell PDMS within the time frame of our experiments.

The LSCM used was a Leica TCS SP8 based on an inverted optical microscope. The LSCM allowed us to record 3D images. However, for an increased time resolution, we did not record full 3D images but rather cross sections of the evaporating water drop on top of the pillar array. The horizontal cross sections $(x y)$ are parallel to the circular sections of the pillars, while the vertical $(x z)$ cross sections are perpendicular to them. The acquisition time of each cross section (3.5 s) was shorter compared to the evaporation timescale of the water drops (40 min).

For imaging the $x y$ and $x z$ cross sections in the infused case, we used a $20 \times / 0.75$ multi-immersion (water) objective, while for the dry case, we used a $40 \times / 0.85$ dry objective. The refractive indices of the different materials are $1.50,1.43,1.29$, and 1.33 for glass, PDMS, ${ }^{32}$ FC-40, and water, respectively. Therefore, FC-40 acts as an index matching liquid when infusing the PDMS pillar array, improving the image quality in these experiments. A combination of 458,476 , and $633 \mathrm{~nm}$ laser excitation lines were used. Besides from brightfield top- 
view images, three different signals were recorded simultaneously. The signals were reflection and two fluorescence signals. The reflected light, highest at the interfaces with a large change in refractive indices, was measured with a photomultiplier (PMT) detector in the range of 455-460 $\mathrm{nm}$. The fluorescence of the Alexa-dyed water and the coumarin-dyed pillars were measured with two different PMTs in the ranges of $690-750$ and $490-555 \mathrm{~nm}$, respectively.

For each experiment, a $4 \mu \mathrm{L}$ Alexa-dyed water drop was manually deposited on top of the pillar array. For the experiments with FC-40, the sample was infused before with FC-40 by depositing the oil and tilting the substrate. Prior to the experiment, the height of the FC-40 layer was similar to that of the pillars; this was checked with the reflection signal from $x z$ cross sections. The sample was surrounded by a ring-shaped container with an optical access from the top to minimize any airflow from the surroundings. The evaporating drop was imaged by the LSCM at approximately $24{ }^{\circ} \mathrm{C}$ and a relative humidity of $40 \%$.

\section{RESULTS}

Cassie-to-Wenzel Transition of an Evaporating Drop on an FC-40-Infiltrated Pillar Array. A time series of a drop evaporating on top of an FC-40-infiltrated PDMS pillar array is shown in Figure 1. Each image is a composite of the grayscale

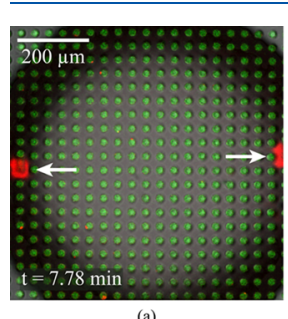

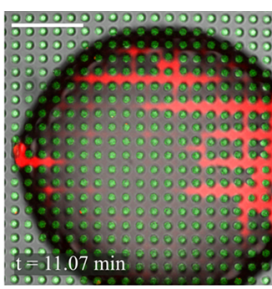

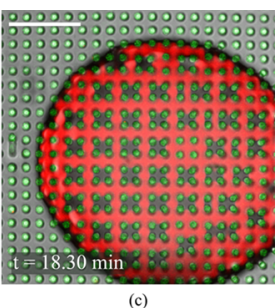

Figure 1. Time series of an evaporating drop on a PDMS pillar array infused with FC-40 (Movie S1). The images are overlays of the confocal images taken $1 \mu \mathrm{m}$ below the pillar top faces. The grayscale represents the brightfield top-view images, $x y$ fluorescence cross sections from PDMS pillars are shown in green, and water is shown in red. The darker regions of the grayscale image are those covered by water, and red regions indicate impalement of water by at least $1 \mu \mathrm{m}$. The FC-40 is not visible. (a) Onset of water impalement, signaled by white arrows, (b) propagation and first bundling events, and (c) formation of regular bundles of pillars. By the last stage, the drop is in a complete Wenzel (impaled) state.

top-view image and both fluorescence signals from the drop (red) and the PDMS pillars (green). When the water drop evaporates, its Laplace pressure increases as its radius decreases. In our experiments, the water drop impales when its radius reaches $370 \pm 47 \mu \mathrm{m}$. We can identify the onset of impalement as the red signal when the Alexa-dyed water starts to become visible on the horizontal cross sections imaged $1 \mu \mathrm{m}$ below the pillar tops. The first impalement sites between pillars are indicated by white arrows in Figure 1a.

Impalement starts at the rim and propagates along either direction of the array. Once a certain gap had been impaled, water did not impale its consecutive parallel gap. Rather, the second one in line is impaled (Figure 1b). The reason for which a line is skipped is the bending of the pillars, as will be demonstrated below. During the impalement process, FC-40 is displaced by the water and the neighboring pillars bend. When the drop has impaled completely, most of the pillars in contact with it display the same four-pillar bundle geometry (Figure 1c). Eventually, when the water has completely evaporated, some of the bundles open up while some others remain in place. Therefore, even if the bundling is triggered, the bundles can still return to upright pillars, depending on the pillar-topillar adhesion. Note that, in contrast to other elasto-capillary problems, the water has completely evaporated at the end of the process so that sticking is only due to adhesion between pillars.

The onset of the impalement transition and symmetry breaking occurs simultaneously. However, they relate two different processes: (i) the transition from the Cassie-toWenzel state (impalement) and (ii) the breaking of symmetry of the initially regular array of pillars. When the water impales the surface, the wetting transition is triggered; the local contact angle is higher than the advancing local contact angle usually measured between the liquid and the surface with a goniometer. However, the impalement transition is independent from the symmetry breaking. The symmetry breaking involves the local contact angle between the water meniscus and the vertical wall of the PDMS micropillar as it influences the capillary force on the micropillar. Notice that the pillars only bend when the capillary force pulling at the pillar top is stronger than its restoring elastic force. Here, we focus on the symmetry breaking that leads the pillars to bundle.

To reveal the assembly mechanism of the bundles, we recorded vertical cross sections at the rim of the water drop (blue plane in Figure 2a). The FC-40-infused regions are

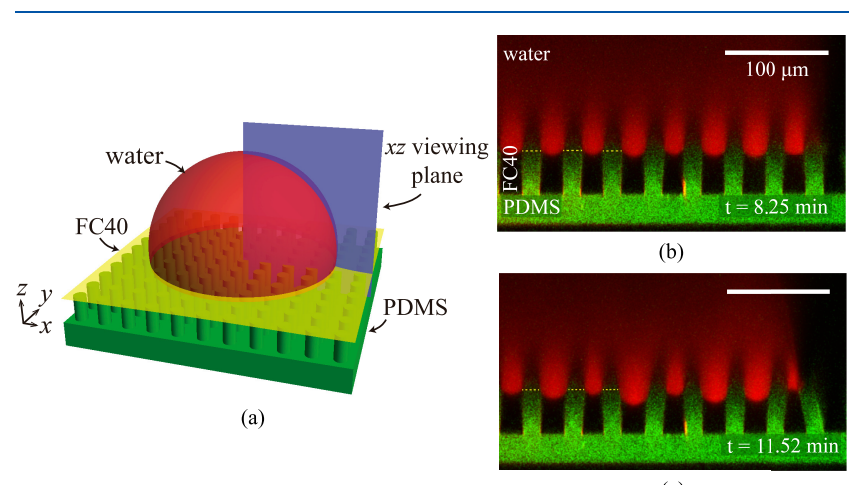

Figure 2. Vertical confocal cross sections of the FC-40-infiltrated structures as the PDMS pillars bundle (Movie S2). The blue cross section in the sketch (a) indicates the position at which (b) and (c) are recorded. In (b), the onset of the symmetry breaking takes place. The local menisci are all similar to one another. (c) shows the spontaneous symmetry breaking as the menisci between each pair of pillars changes. Deeper (shallower) menisci are present when the pillars bend away (toward) from each other.

represented in black, while the fluorescence signals from the drop and the PDMS pillars are red and green, respectively (Figure $2 b, c)$. In the vertical cross sections, optical artefacts are visible over the pillars. Scattering of light causes a decrease in the intensity of the detected light. Thus, for clarity, we have marked the approximate position of some of the pillar top faces by yellow dashed lines.

At the onset of symmetry breaking, below the drop, only the pillar directly at the edge of the drop was slightly bent inward (rightmost pillar in Figure 2b). As evaporation progresses, pairs of pillars bend toward or away from each other, further breaking the symmetry of the structure (Figure 2c and Movie S2). The experiments show a correlation between the deformation of the pillars and the shape of the liquid menisci such that where the meniscus was deeper (shallower), the pillar spacing became wider (narrower). This observation 
shows that bundling is a collective process. The bending of one pillar influences the bending of its neighbors via the capillary interaction mediated by water.

In the experiments, bundling always started from the outer edge of the water drop or near a defect in the pillar array. Slight deviations from the mean spacing between pillars act as a perturbation to the symmetry of the array and cause the water to start penetrating between the pillars. Then, the pillars bend away from each other allowing the water surface to bulge even lower. As a result, the gap between the pillars widens, which facilitates further penetration of water. Ultimately, the process ends in a complete Wenzel state.

Horizontal and vertical images (Figures 1 and 2) demonstrate that bundles of pillars are formed while individual water menisci between pairs of pillars infiltrate the surface. Bundling is due to a spontaneous symmetry breaking underneath the water drop leading to a collective impalement pattern.

Cassie-to-Wenzel Transition in Air. The effect of replacing FC-40 with air was twofold: First, the interpillar menisci were flatter than in FC-40-infused arrays; the contact angle of the water menisci on the PDMS pillars was around $90^{\circ}$ (Figure 3). Second, no significant pillar bending was

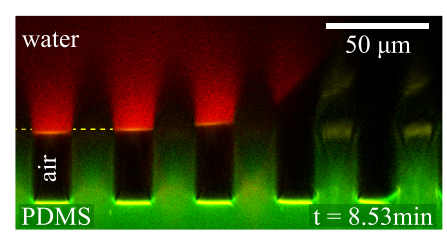

Figure 3. Vertical cross-section of a water drop (red) on a superhydrophobic pillar array surface (green) at the onset of the Cassie-to-Wenzel transition (Movie S4). The water-air and PDMSair interfaces are visible as reflections (shown in yellow) due to the high difference in refractive index between water and air.

observed. We observed impalement but not symmetry breaking of consecutive menisci. There was no bundling of the micropillars during the impalement of water, showing that bundling and impalement are different processes. In air, the Cassie-to-Wenzel transitions either led to a complete filling of the substrate by water or to the formation of an air pocket (Movie S3). The air pockets agree with those observed by Papadopoulos et at.; ${ }^{33}$ they were only slightly larger as our pillars $(40 \mu \mathrm{m})$ were taller than theirs $(25 \mu \mathrm{m})$.

\section{MECHANISM OF SYMMETRY BREAKING}

We now wish to explain the mechanism of symmetry breaking: the onset of bundling. For this, we consider the two competing effects: capillarity and elasticity. From the capillary perspective, having alternating deep and shallow menisci is energetically more favorable as compared to having two symmetric menisci. Elasticity plays the reverse role of capillarity since it opposes deformation. A similar balance was used to describe the sticking condition, predicting the condition for bundles to stay together. ${ }^{10,14,17}$ Here, we rather focus on the symmetry breaking of the initially regular periodic array of pillars, the reason why the pillars start to bend in the first place. In what follows, we explain the mechanism of symmetry breaking in terms of the forces rather than in terms of the energy, which is discussed in the Appendix.

We develop a simplified model where we consider the balance between capillary and elastic forces. Inspired by the analysis of Hadjittofis et al., ${ }^{7}$ we consider a simplified setting; the complex system of periodic three-dimensional pillars is reduced to a set of two-dimensional pillars of height $h$ and center-to-center spacing $w$ (Figure $4 \mathrm{a}$ ). In the model, the outer
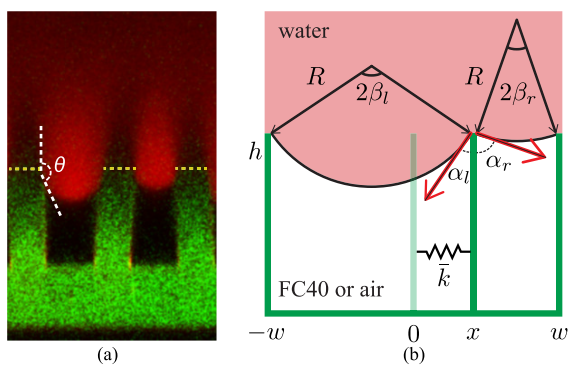

Figure 4. (a) Fraction of the water drop's interface with the PDMS pillars from Figure $2 \mathrm{~b}$. (b) Sketch of the two-dimensional model representing the experiment. The flexibility of the pillars is represented by a moving central pillar connected to its original position by a linear elastic spring with a spring constant $\bar{k}$. The effect of capillarity is accounted for by the contact line forces, indicated by red arrows.

pillars are kept fixed in place while the central one can be displaced laterally, mimicking the flexible behavior of the real pillars by a linear spring connected to the central position. The displacement is denoted $x . \bar{k}$ is the equivalent elastic constant of the pillars in the two-dimensional case, $\bar{k}=k / w\left([\mathrm{~N}] /\left[\mathrm{m}^{2}\right]\right)$, and capillarity is accounted for by the menisci (radius $R$ ) in between the pillars.

Importantly, at the onset of the symmetry breaking in the experiment (Figure 2a), the contact line is still pinned to the pillar tops. Therefore, the sidewalls in Figure $4 \mathrm{~b}$ remain fully wetted by the liquid below as long as impalement does not occur. Impalement occurs only when the static advancing angle of the top liquid is reached. Thus, as long as the contact angle on the pillar does not exceed this static advancing angle, the point force can still be considered to act at the top of the pillar. With this, the total horizontal force per unit length on the central pillar reads

$$
\begin{aligned}
F_{x} & =-\bar{k} x+\gamma\left[\sin \alpha_{r}-\sin \alpha_{l}\right] \\
& =-\bar{k} x+\gamma\left[\sqrt{1-\left[\frac{w-x}{2 R}\right]^{2}}-\sqrt{1-\left[\frac{w+x}{2 R}\right]^{2}}\right]
\end{aligned}
$$

Here, we have used $\alpha_{l, r}=\pi / 2-\beta_{l, r}$ with $\sin \beta_{l, r}=\frac{w \pm x}{2 R}$, given by the geometry sketched in Figure $4 \mathrm{~b}$. With this force, we will see below that the effect of capillarity is to promote the breaking of symmetry, while the effect of elasticity is to resist it.

First, we focus on the linear range of the force as we are most interested in the onset of the symmetry breaking. Then, we discuss the non-linear response of the system. While below, we discuss the results in terms of forces; the equivalent energetic derivation is provided in the Appendix.

Linear Regime. We start by considering small deformations of the central pillar. For this, we linearize eq 2 around $x=$ 0

$$
F_{x} \approx\left[-\bar{k}+\frac{\gamma w}{R \sqrt{4 R^{2}-w^{2}}}\right] x
$$


The opposite signs of the terms on the right nicely illustrate the opposing characters of capillarity and elasticity. The linearized form of the force, eq 3 , is such that when elasticity is dominant, its prefactor is negative. In that case, the resultant force is restoring, having the opposite sign of the displacement, which renders $x=0$ a stable equilibrium. In contrast, when capillarity is dominant, the prefactor is positive. Then, the total force has the same sign as the displacement such that the symmetric situation where $x=0$ becomes unstable. Thus, the criteria for spontaneous symmetry breaking is $-\bar{k}+\frac{\gamma w}{R \sqrt{4 R^{2}-w^{2}}} \geq 0$ or, in terms of the contact angle $(\theta)$

$$
\frac{\cos \theta}{|\tan \theta|} \leq-\frac{\bar{k} w}{2 \gamma}
$$

where we have used $\theta=\pi / 2+\arcsin (w / 2 R)$. In terms of the material properties of the pillars, this gives a critical contact angle $\theta_{\mathrm{c}}$ defined by

$$
\frac{\cos \theta_{c}}{\left|\tan \theta_{c}\right|}=-\frac{3 \pi}{128} \frac{E d^{4}}{\gamma h^{3}}
$$

Interestingly, the critical value of the contact angle is independent of the pillar-to-pillar distance. This is because the breaking of symmetry appears at the onset of bundling, where the pillar deflection is initially infinitesimal: whether or not an initial perturbation is amplified depends only on the projection of the capillary force and does not involve the spacing. This is in stark contrast with the sticking conditions discussed in Py et al. ${ }^{10}$ and Bico et al. ${ }^{14}$ In that case, the deflection of the pillars is decisive, and hence the distance between the pillars appears as a key parameter.

We remark that, in the model, the contact line is assumed to remain pinned at the top of the pillar. In reality, the contact line will move once the liquid's contact angle exceeds the static advancing contact angle $\left(\theta_{\mathrm{a}}\right)$. Whether or not symmetry breaking occurs prior to impalement, therefore, depends on the magnitude of $\theta_{\mathrm{a}}$ and $\theta_{\mathrm{c}}$. If $\theta_{\mathrm{a}}>\theta_{\mathcal{c}}$ the symmetry breaking occurs prior to impalement. On the other hand, if $\theta_{\mathrm{a}}<\theta_{\mathcal{c}}$ the liquid will fill the gaps of the array without triggering symmetry breaking.

In practice, since the pressure inside the drop is positive, we only need to consider $\theta \geq 90^{\circ}$. From eq 4 , we then infer that the system will typically be below the threshold of symmetry breaking for angles close to $90^{\circ}$, even for very soft pillars. However, the increase of $\theta$ due to the increase of pressure inside the drop could indeed cause a change in sign in eq 4 and hence a breaking of symmetry.

The proposed mechanism for symmetry breaking, associated with a large contact angle of the menisci, is indeed supported by our experiments. We measured the maximum contact angles (the advancing contact angles, $\theta_{\mathrm{a}}$ ) from the vertical cross sections for the non-bundling and bundling cases. These are $91^{\circ}$ for air and $131^{\circ}$ for FC-40. So indeed, for the air-filled case, the interface remains almost perpendicular to the pillar walls throughout the impalement process and bending is not observed. By contrast, the FC-40-infused case exhibits large bulges and bending is observed.

Despite the simplifying assumptions of the model, we can go one step further and quantitatively estimate the stability threshold, $\theta_{\mathcal{c}}$, corresponding to both the FC-40 and air-filled surfaces. The spring constant, $\bar{k}$, is the same for both cases with $k \approx 200 \mathrm{mN} / \mathrm{m}$ and $w=40 \mu \mathrm{m}$, while the surface tensions are slightly different. Therefore, eq 5 gives slightly different values for the critical angle, namely, $\theta_{c}^{F C 40} \approx 144^{\circ}$ for the FC-40infused surface, and $\theta_{\mathrm{c}}^{\text {air }} \approx 139^{\circ}$ for the air-filled surface. Therefore, the maximum contact angle mentioned before for FC-40 $\left(\theta_{\mathrm{a}}=131^{\circ}\right)$ is indeed close to the predicted onset $\left(\theta_{\mathrm{c}}=\right.$ $\left.144^{\circ}\right)$ as opposed to the air-filled menisci $\left(\theta_{\mathrm{a}}=91^{\circ}\right)$, which are too shallow to cause any symmetry breaking, in line with the proposed mechanism for bundling.

Nonlinear Regime. We now examine the predictions of the model in the nonlinear regime where the displacement $x$ no longer remains small. Interestingly, it is necessary to distinguish between two possible scenarios, depending on whether the impalement occurs at constant pressure or at constant volume. In the first scenario, we assume that the pressure inside the lubricant layer (FC-40 or air) is the same as that of the surrounding air and thus is kept constant as the system equilibrates. In this case, the value of $R$ appearing in eq 2 follows from the imposed value of $\theta$. In the second scenario, we assume that the equilibration of $x$ occurs at a constant volume, e.g., the transport of lubricant induces a pressure difference with respect to the air pressure. In that case, $R$ has to be treated as a function of $x$ for the imposed lubricant volume, which in our two-dimensional model is actually the total area of the menisci. This relation is obtained numerically, as explained in the Appendix.

Figure 5 shows the nonlinear force-displacement curves for typical examples (a) below the threshold of symmetry breaking

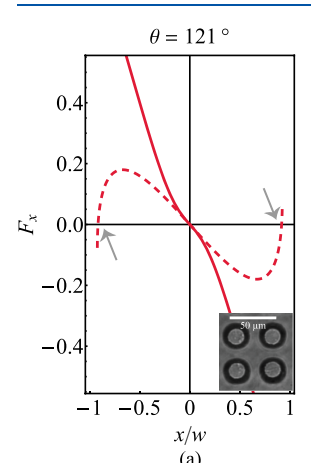

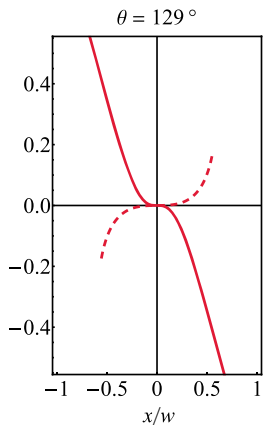

(b)

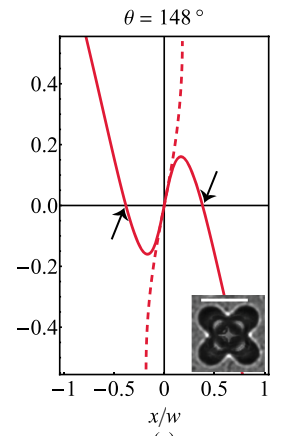

(c)
Figure 5. Force on the central pillar calculated assuming a constant area (solid line), and constant pressure (dashed line), assuming for convenience, $\bar{k} w / \gamma=1$. Notice that both calculations coincide in the linear regime $(x \rightarrow 0)$. (a) Below, (b) at and (c) above the critical contact angle $\theta_{\mathrm{c}}=129^{\circ}$.

$\left(\theta<\theta_{c}\right)$, (b) at the threshold of symmetry breaking $\left(\theta=\theta_{\mathrm{c}}\right)$, and (c) above the threshold of symmetry breaking $\left(\theta>\theta_{c}\right)$. This is achieved by assuming $\bar{k} w / \gamma=1$ and increasing the value of $\theta$. Indeed, we see that the two approaches, constant pressure (dashed line) and constant area (solid line), give different results.

As expected, we find a transition between a stable and an unstable spring at the threshold value calculated with eq $5, \theta_{c}$ $\approx 129^{\circ}$ (Figure $5 \mathrm{~b}$ ). This transition is illustrated by comparing the slope of the force in the vicinity of $x=0$ for different contact angles. Below the threshold (Figure 5a), this slope is negative, while above the threshold (Figure $5 \mathrm{c}$ ), it is positive. A negative slope yields no spontaneous symmetry breaking as any deformation from the spring is counteracted by the elastic force. The contrary is true for a positive slope where symmetry breaking is expected since any spring deformation will be amplified. 
Both approaches, constant area and pressure, show the predicted symmetry breaking. However, we find two different types of transition since we find different stable and unstable equilibria for each approach. Below the critical contact angle $(\theta$ $<\theta_{\mathrm{c}}=129^{\circ}$ ), the constant area approach (solid line in Figure 5a) shows only one stable equilibrium at $x=0$; the force and displacement have opposite signs. In contrast, the constant pressure approach shows one stable equilibrium $(x=0)$ and two unstable equilibria as $x \rightarrow w$ (gray arrows in Figure 5a). Therefore, the symmetric configuration with upright pillars (inset Figure 5a) is expected in this case since any other configuration would be unstable.

Above the critical contact angle $\left(\theta>\theta_{c}\right.$, Figure 5c), the constant area approach shows two new stable equilibria (dark arrows). In contrast, the constant pressure approach shows only unstable configurations; the force and displacements always have the same sign.

For the constant area approach, the transition toward bending is continuous, and for the constant pressure approach, it is discontinuous. In the first case, when the contact angle grows larger, we identify new stable equilibria like in a secondorder phase transition. In the second case, there are no new stable equilibria and the spring goes from being stable to unstable like in a first-order phase transition. The value of $x$ jumps to its maximum value immediately above the threshold of symmetry breaking. In experiments, when the pillars are infused with FC-40, we see that the pillars deform slowly to form the bundles (Figure 4a and Movie S2). This gradual shift from upright to bundled pillars suggests a continuous transition with stable equilibria different than $x=0$, in line with the constant area approach. However, it can also be the case that, in the quasi-static evolution of the system, the evaporating drop imposes a pressure jump across the interface and, if the pressure in the bottom liquid remains constant (as in the air case), the transition would be discontinuous (firstorder).

\section{CONCLUSIONS}

We studied the formation of bundles of soft, hydrophobic micropillars under an evaporating water drop. A comparison between the Cassie-to-Wenzel transition in air and FC40 shows that the local contact angle is fundamental for the onset of the bundling process. We observe that bundling starts as a spontaneous breaking of symmetry in the consecutive menisci below the water drop. Experimentally, a point defect, e.g., a missing pillar in the array, slight shifts in pillar positions, or the bending of the pillar at the rim of the drop, serves as an initial perturbation of the meniscus symmetry, which is later on amplified by the interplay between capillary and elastic forces.

We find that a fundamental parameter in the onset of the symmetry breaking is $\theta$, the local contact angle of the liquid with respect to the pillar wall. As the water-PDMS contact angle becomes higher, the pillar array becomes mechanically more unstable and likely to bundle. We propose a simple twodimensional model where the breaking of symmetry emerges from a balance of capillarity and elasticity. In particular, we recover that the symmetry breaking requires large contact angles, in good agreement with the experiments. Interestingly, in the model, the behavior above the threshold can be continuous or discontinuous. That is, the pillar deformation can show new local equilibria or suffer a sharp transition from upright to bent. Experimentally, the transition is continuous, which suggests that the pressure inside the lubricant is not in quasi-static equilibrium with the air pressure according to our model.

The model consists of only three consecutive pillars rather than a periodic array. Therefore, a natural path for the following research is to incorporate the meniscus contact angle into more elaborate models with more than three pillars and the possibility of pillar stiction to predict larger bundles of taller (or softer) pillars. However, the simple model is sufficient for the purpose of describing the symmetry breaking of the initially periodic pattern. Indeed, the essential ingredients in the simple spring-pillar model reproduce the symmetry breaking of the real PDMS micropillars and predict a threshold contact angle in good qualitative agreement with our experimental results.

\section{APPENDIX}

In the main text, we presented the mechanism of symmetry breaking in the context of the forces involved. However, this symmetry breaking can also be analyzed in terms of the energy of the system. In this appendix, we will show the energy-based approach and will find that both force and energy approaches are equivalent. Therefore, we consider the energy per unit length of the system as the sum of the capillary and elastic energies and, in the case of constant pressure, add the work done on the top liquid $(W=-\delta P A)$. With this, the energy of the system as the liquid infiltrates into the surface reads

$$
E=2 \gamma R\left[\sin ^{-1}\left(\frac{w-x}{2 R}\right)+\sin ^{-1}\left(\frac{w+x}{2 R}\right)\right]+\frac{1}{2} \bar{k} x^{2}+W
$$

The first two terms of the equation are related to the length of the menisci, while the third accounts for the energy stored by deforming the spring connected to the central pillar.

As noted previously, we examine two scenarios in the quasistatic evolution of the system: an isobaric and an isochoric process. In the first scenario, the work done by the pressure is non-zero as the liquid area that sags into the interpillar gaps is not fixed. In the second scenario, the liquid area sagging into the gaps is the control variable. Keeping the area constant implies that the total work is zero and, therefore, the equation is reduced to two contributions, capillarity and elasticity.

For either approach, we must compute the area of the sagging menisci (instead of the volume since this is a twodimensional model). For this, we compute the sum of the area of two circular segments of radius $R$ as sketched in Figure $4 \mathrm{~b}$.

$$
\begin{aligned}
A= & R^{2}\left[\sin ^{-1}\left(\frac{w-x}{2 R}\right)+\sin ^{-1}\left(\frac{w+x}{2 R}\right)\right. \\
& -\frac{w-x}{2 R} \sqrt{1-\left[\frac{w-x}{2 R}\right]^{2}} \\
& \left.-\frac{w+x}{2 R} \sqrt{1-\left[\frac{w+x}{2 R}\right]^{2}}\right]
\end{aligned}
$$

For the isochoric process, we find the radius of the meniscus as a function of the area by numerically solving eq 7 via Newton's method. The same method was used to calculate $R(x, A)$ for the force curves presented in Figure 5. Equation 7 was solved under the constraint $0 \leq A \leq 0.79$ where the maximum value of this range was computed as the area at zero deformation and a minimum possible radius; $x=0$ and $R=\frac{w}{2}$. Notice that, if $x=0$ and the liquid is pinned at the top of the 
pillars, the radius of the meniscus is strictly larger than half the separation between the consecutive pillars; $R \geq \frac{w}{2}$. For the isobaric process, we compute the total energy (with $W \neq 0$ ), assuming the radius of the meniscus to be a constant such that $R \geq \frac{w}{2}$.

The predictions of the model are now presented in the form of an energy landscape in Figure 6 for both isochoric (solid lines) and isobaric scenarios (dashed lines). The force diagram of Figure 5 is simply recovered by the connection $F=-\mathrm{d} E / \mathrm{d} x$.

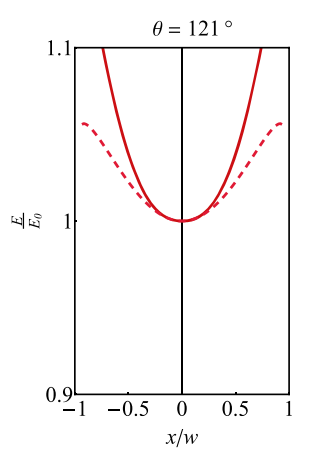

(a)

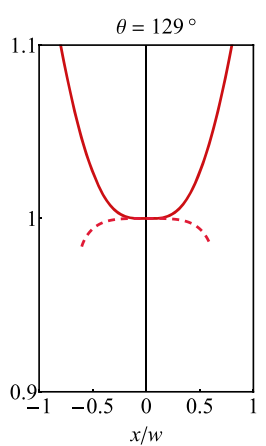

(b)

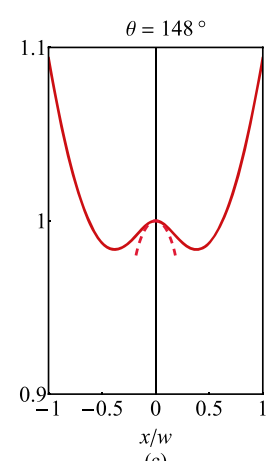

(c)
Figure 6. Energy calculated at a constant area (solid lines), and constant pressure (dashed lines) for increasing values of contact angle. The calculations were performed considering $\bar{k} w / \gamma=1$. (a) Below, (b) at and (c) above the critical contact angle $\theta_{c}=129^{\circ}$.

As in the force case, the transition from stable to unstable (Figure $6 \mathrm{~b}$ ) is found at the critical value calculated with eq 4, $\theta$ $=129^{\circ}$. Below the transition (Figure 6a), both the isochoric and isobaric scenarios are stable at $x=0$. This is illustrated by the concave energy landscape with a minimum at $x=0$. Above the transition (Figure 6c), the energy landscape is convex with a local maximum at $x=0$. As anticipated by the force approach, we find two types of transitions. The isobaric scenario yields a first-order transition where the energy changes from a concave to a convex landscape at the transition value given by eq 4 . In contrast, the isochoric scenario yields a second-order transition where the energy landscape changes to a double-well configuration, creating two new stable states at $x$ $\neq 0$.

\section{ASSOCIATED CONTENT}

\section{SI Supporting Information}

The Supporting Information is available free of charge at https://pubs.acs.org/doi/10.1021/acs.langmuir.0c02147.

Movie S1: Cassie-to-Wenzel transition of an evaporating drop on an FC-40-infiltrated pillar array and overlays of a top-view image and both $x y$ fluorescence cross sections from PDMS pillars and water (AVI)

Movie S2: Time series of the vertical confocal cross sections of the FC-40 infiltrated structures as the PDMS pillars bundle (AVI)

Movie S3: Cassie-to-Wenzel transition of an evaporating drop on a pillar array (no lubricant layer) (AVI)

Movie S4: Time series of the vertical confocal cross sections at the onset of the Cassie-to-Wenzel transition in the air-filled experiments (AVI)

\section{AUTHOR INFORMATION}

\section{Corresponding Author}

Hans-Jürgen Butt - Max Planck Institute for Polymer Research, Mainz 55128, Germany; 이이.org/0000-0001-53912618; Phone: +49 6131379 111; Email: butt@mpipmainz.mpg.de; Fax: +496131379310

\section{Authors}

Diana Garcia-Gonzalez - Max Planck Institute for Polymer Research, Mainz 55128, Germany; Physics of Fluids group, Max-Planck Center Twente for Complex Fluid Dynamics, Department of Science and Technology, University of Twente, Enschede 7500 AE, Netherlands; 이이.org/0000-00025170-0993

Jacco Snoeijer - Physics of Fluids group, Max-Planck Center Twente for Complex Fluid Dynamics, Department of Science and Technology, University of Twente, Enschede 7500 AE, Netherlands

Michael Kappl - Max Planck Institute for Polymer Research, Mainz 55128, Germany; 이이이.org/0000-0001-7335-1707

Complete contact information is available at:

https://pubs.acs.org/10.1021/acs.langmuir.0c02147

\section{Notes}

The authors declare no competing financial interest.

\section{ACKNOWLEDGMENTS}

We are grateful to M. D'Acunzi, A. Kaltbeitzel, P. Papadopoulos, B.E. Pinchasik, and W.S.Y. Wong for their experimental insights as well as to S.G. Huisman and V. Sanjay for various stimulating discussions. We acknowledge the support of the Max Planck Center for Complex Fluid Dynamics - University of Twente.

\section{REFERENCES}

(1) Butt, H.-J.; Roisman, I. V.; Brinkmann, M.; Papadopoulos, P.; Vollmer, D.; Semprebon, C. Characterization of super liquid-repellent surfaces. Curr. Opin. Colloid Interface Sci. 2014, 19, 343-354.

(2) Lafuma, A.; Quéré, D. Superhydrophobic states. Nat. Mater. 2003, 2, 457.

(3) Cassie, A. B. D.; Baxter, S. Wettability of porous surfaces. Trans. Faraday Soc. 1944, 40, 546-551.

(4) Tanaka, T.; Morigami, M.; Atoda, N. Mechanism of Resist Pattern Collapse during Development Process. Jpn. J. Appl. Phys. 1993, 32, 6059-6064.

(5) Tanaka, T.; Morigami, M.; Oizumi, H.; Soga, T.; Ogawa, T.; Murai, F. Prevention of resist pattern collapse by resist heating during rinsing. J. Electrochem. Soc. 1994, 141, L169-L171.

(6) Neukirch, S.; Roman, B.; de Gaudemaris, B.; Bico, J. Piercing a liquid surface with an elastic rod: Buckling under capillary forces. $J$. Mech. Phys. Solids 2007, 55, 1212-1235.

(7) Hadjittofis, A.; Lister, J. R.; Singh, K.; Vella, D. Evaporation effects in elastocapillary aggregation. J. Fluid Mech. 2016, 792, 168185.

(8) Chandra, D.; Yang, S. Capillary-Force-Induced Clustering of Micropillar Arrays: Is It Caused by Isolated Capillary Bridges or by the Lateral Capillary Meniscus Interaction Force? Langmuir 2009, 25, 10430-10434.

(9) Chandra, D.; Yang, S. Stability of High-Aspect-Ratio Micropillar Arrays against Adhesive and Capillary Forces. Acc. Chem. Res. 2010, 43, 1080-1091.

(10) Py, C.; Bastien, R.; Bico, J.; Roman, B.; Boudaoud, A. 3D aggregation of wet fibers. Europhys. Lett. 2007, 77, 44005.

(11) Kim, H.-Y.; Mahadevan, L. Capillary rise between elastic sheets. J. Fluid Mech. 2006, 548, 141-150. 
(12) Duprat, C.; Protière, S.; Beebe, A. Y.; Stone, H. A. Wetting of flexible fibre arrays. Nature 2012, 482, 510.

(13) Duprat, C.; Aristoff, J. M.; Stone, H. A. Dynamics of elastocapillary rise. J. Fluid Mech. 2011, 679, 641-654.

(14) Bico, J.; Reyssat, É.; Roman, B. Elastocapillarity: When Surface Tension Deforms Elastic Solids. Annu. Rev. Fluid Mech. 2018, 50, 629-659.

(15) Roman, B.; Bico, J. Elasto-capillarity: deforming an elastic structure with a liquid droplet. J. Phys.: Condens. Matter 2010, 22, 493101.

(16) Pokroy, B.; Kang, S. H.; Mahadevan, L.; Aizenberg, J. SelfOrganization of a Mesoscale Bristle into Ordered, Hierarchical Helical Assemblies. Science 2009, 323, 237-240.

(17) Bico, J.; Roman, B.; Moulin, L.; Boudaoud, A. Elastocapillary coalescence in wet hair. Nature 2004, 432, 690-690.

(18) De Volder, M.; Hart, A. J. Engineering hierarchical nanostructures by elastocapillary self-assembly. Angew. Chem., Int. Ed. 2013, 52, 2412-2425.

(19) Tawfick, S. H.; Bico, J.; Barcelo, S. Three-dimensional lithography by elasto-capillary engineering of filamentary materials. MRS Bull. 2016, 41, 108-114.

(20) Kang, S. H.; Pokroy, B.; Mahadevan, L.; Aizenberg, J. Control of Shape and Size of Nanopillar Assembly by Adhesion-Mediated Elastocapillary Interaction. ACS Nano 2010, 4, 6323-6331.

(21) Duan, H.; Berggren, K. K. Directed Self-Assembly at the $10 \mathrm{~nm}$ Scale by Using Capillary Force-Induced Nanocohesion. Nano Lett. 2010, 10, 3710-3716.

(22) De Volder, M.; Tawfick, S. H.; Park, S. J.; Copic, D.; Zhao, Z.; Lu, W.; Hart, A. J. Diverse 3D Microarchitectures Made by Capillary Forming of Carbon Nanotubes. Adv. Mater. 2010, 22, 4384-4389.

(23) Chandra, D.; Yang, S.; Soshinsky, A. A.; Gambogi, R. J. Biomimetic Ultrathin Whitening by Capillary-Force-Induced Random Clustering of Hydrogel Micropillar Arrays. ACS Appl. Mater. Interfaces 2009, 1, 1698-1704.

(24) Taroni, M.; Vella, D. Multiple equilibria in a simple elastocapillary system. J. Fluid Mech. 2012, 712, 273-294.

(25) Chiodi, F.; Roman, B.; Bico, J. Piercing an interface with a brush: Collaborative stiffening. Europhys. Lett. 2010, 90, 44006.

(26) Singh, K.; Lister, J. R.; Vella, D. A fluid-mechanical model of elastocapillary coalescence. J. Fluid Mech. 2014, 745, 621-646.

(27) Qin, D.; Xia, Y.; Whitesides, G. M. Soft lithography for microand nanoscale patterning. Nat. Protoc. 2010, 5, 491-502.

(28) Goodno, B.; Gere, J. Mechanics of Materials; SI Edition; Cengage Learning: 2017.

(29) Papadopoulos, P.; Pinchasik, B.-E.; Tress, M.; Vollmer, D.; Kappl, M.; Butt, H.-J. Wetting of soft superhydrophobic micropillar arrays. Soft Matter 2018, 14, 7429-7434.

(30) Johnson, K. L.; Kendall, K.; Roberts, A. D. Surface energy and the contact of elastic solids. Proc. R. Soc. London, Ser. A 1971, 324, 301-313.

(31) Johnson, K. L. Contact Mechanics; Cambridge University Press: 1985

(32) Schneider, F.; Draheim, J.; Kamberger, R.; Wallrabe, U. Process and material properties of polydimethylsiloxane (PDMS) for Optical MEMS. Sens. Actuators, A 2009, 151, 95-99.

(33) Papadopoulos, P.; Mammen, L.; Deng, X.; Vollmer, D.; Butt, H.-J. How superhydrophobicity breaks down. Proc. Natl. Acad. Sci. U. S. A. 2013, 110, 3254-3258. 